\title{
Tumores de Cólon - Primeiro Achado do Adenocarcinoma de Pâncreas: Relato de Caso
}

\author{
Colon Tumors - First find of the Pancreatic Adenocarcinoma: Case Report
}

\author{
SANDRAPEDROSO DE MORAES ${ }^{1}$; FRANCISCO CLARO JÚNIOR²; JOAQUIM JOSÉ DE OLIVEIRAFILHO'; GUSTAVO \\ SEVÁ-PEREIRA ${ }^{1} ;$ RICARDO BOLZAM DO NASCIMENTO $^{1}$, AMILCAR DECASTRO $^{3}$
}

1. Médico do serviço de coloproctologia do HMMG; ${ }^{2}$. Residente do serviço de coloproctologia do HMMG; ${ }^{3 .}$ Médico patologista da Pontifícia Universidade Católica de Campinas.

\begin{abstract}
MORAES SP; CLARO JÚNIOR F; OLIVEIRA FILHO JJ; SEVÁ-PEREIRA G; NASCIMENTO RB; CASTRO A. Tumores de Cólon Primeiro Achado do Adenocarcinoma de Pâncreas: Relato de Caso. Rev bras Coloproct, 2007;27(3): 322-327.

RESUMO: OBJETIVO: Relatar um caso raro de adenocarcinoma de pâncreas que se apresentou como tumores colorretais sincrônicos. Paciente masculino, 76 anos, apresentava dor abdominal difusa de forte intensidade, diarréia e vômitos há sete dias. Tratava de gastrite há dois anos e nos últimos quatro meses apresentava hiporexia e perda de peso. Estava emagrecido, desidratado e desnutrido, com distensão abdominal importante, ruídos hidroaéreos ausentes e dor difusa à palpação abdominal. Exames evidenciaram hiperglicemia, distensão importante do intestino delgado ao raio $\mathrm{x}$, ultra-som de abdome com colecistolitíase $\mathrm{e}$ endoscopia digestiva alta com pangastrite, bulboduodenite e papila normal. Tomografia abdominal confirmou colecistolitíase. A colonoscopia mostrou três lesões, em reto médio, cólon transverso e na válvula íleocecal. As biópsias revelaram apenas reação inflamatória. Persistiram os sintomas e decidiu-se submetê-lo a colecistectomia onde foram vistas lesões planas em diafragma cujas biópsias evidenciaram adenocarcinoma. No quinto dia de pós-operatório o paciente apresentava quadro obstrutivo e foi submetido à nova laparotomia com colectomia direita, ileostomia terminal dupla e biópsia pancreática. Esta mostrou adenocarcinoma e o estudo imunoistoquímico positivo para tumor primário do pâncreas. $O$ paciente evoluiu para óbito um mês após. CONCLUSÃO: o exame de imagem normal não descarta a hipótese diagnóstica e quando a origem do tumor primário não está definida é essencial o exame imunoistoquímico para firmar o diagnóstico.
\end{abstract}

Descritores: neoplasias pancreáticas, neoplasias colorretais não polipóides, metástase neoplásica, adenocarcinoma, tomografia computadorizada por raios $\mathrm{x}$.

\section{INTRODUÇÃO}

Adenocarcinomas sincrônicos avançados ocorrem em 3 a $7 \%$ dos pacientes com carcinoma colorretal ${ }^{1,2}$. A confirmação diagnóstica nem sempre é obtida no primeiro exame anatomopatológico, geralmente por falhas na coleta do material. Biópsias contendo material necrótico ou inflamatório podem falsear o diagnóstico.

\section{OBJETIVO}

Relatar o caso de um paciente com diagnóstico inicial de tumor colorretal sincrônico que na verdade tratava-se de adenocarcinoma de corpo de pâncreas.

\section{RELATO DE CASO}

A.A, 76 anos, masculino, natural de Funchal, Portugal, e procedente do Rio de Janeiro, deu entrada nesse serviço com dor abdominal difusa, tipo cólica, de forte intensidade, diarréia e vômitos há uma semana.

Relatou que há dois anos apresentava depressão, episódios intermitentes e esporádicos de epigastalgia com irradiação para dorso que piorava com a ingestão alimentar, associada a empachamento pósprandial. Por duas vezes foi diagnosticada gastrite com Helicobacter pilory positivo (HP+) realizando tratamento clínico, porém com manutenção dos sintomas, piorando nos últimos quatro meses, quando apresentou

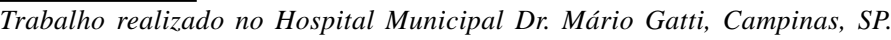

$\overline{\text { Recebido em 15/01/2007 }}$

Aceito para publicação em 21/03/2007 
hiporexia e perda de aproximadamente seis quilogramas.

Ao exame físico, o paciente encontrava-se intensamente desidratado e moderadamente desnutrido, com distensão abdominal importante, ruídos hidroaéreos ausentes e dor difusa à palpação abdominal. À radiografia abdominal apresentava distensão importante do intestino delgado com edema de alças, sem níveis hidroaéreos. Nos exames laboratoriais apresentava alterações de glicemia (137mg\%), com valores crescentes na evolução clínica e VHS $\left(11 \mathrm{~mm} / \mathrm{h} 1^{\text {a }}\right.$ hora e 28 $\mathrm{mm} / \mathrm{h}$ de $2^{\mathrm{a}}$ hora). Trouxe exames recentes de outro serviço mostrando colecistolitíase e cisto renal esquerdo no ultra-som de abdome. Endoscopia digestiva alta apresentando pangastrite moderada, bulboduodenite, papila sem alterações e HP+. O paciente foi tratado clinicamente e os exames foram novamente realizados em nosso hospital e seus resultados confirmados. Prosseguiu-se a investigação com tomografia de abdome com cortes finos para pâncreas e vias biliares que mostrou colecistolitíase, granuloma esplênico, cisto renal esquerdo e aumento prostático (biópsia sem sinais de malignidade). A investigação continuou com colonoscopia que mostrou lesão estenosante de 18 a $8 \mathrm{~cm}$ da margem anal e outras duas lesões menores, em cólon transverso e na válvula íleocecal (ocluindo-a parcialmente). Todas as lesões apresentavam-se de consistência bem endurecida com coloração vinhosa. A avaliação microscópica das biópsias revelou apenas reação inflamatória sem sinais de malignidade. Esta foi repetida mais duas vezes, mantendo o mesmo laudo histopatológico. A persistência de vômitos e dor epigástrica foi, então, atribuída a colecistolitíase e o grupo de urgência e trauma do hospital decidiu submetê-lo a colecistectomia videolaparoscópica. Durante o procedimento foram evidenciadas algumas lesões planas peritoneais, de cor esbranquiçada, em diafragma e área abaulada e endurecida periduodenal, optando-se por conversão para colecistectomia aberta. Na investigação da cavidade foram palpadas as lesões identificadas na colonoscopia, e realizou-se biópsia de lesão diafragmática e de área endurecida na região periduodenal. $\mathrm{O}$ exame histopatológico evidenciou adenocarcinoma bem diferenciado infiltrativo em peritônio e tecido adiposo. Suspeitando-se de tumor primário de cólon, o paciente foi transferido para a enfermaria de coloproctologia. No quinto dia de PO, apresentou piora clínica importante e com hipótese diagnóstica de abdome agudo obstrutivo, secundário à lesão do íleo terminal o paciente foi subme- tido à nova laparotomia com colectomia direita, ileostomia e fístula mucosa. Foi retirado fragmento de pâncreas que encontrava-se endurecido. Este veio como adenocarcinoma, e o estudo imuno-histoquímico positivo para tumor primário do pâncreas, com positividade de citoceratinas (CK) de baixo e alto peso molecular, citoceratinas 7, 19 e 20, antígeno de membrana epitelial (EMA), antígeno carcinoembrionário (CEA) e antígeno associado a câncer gastrointestinal (Ca 19.9). O paciente manteve quadro álgico e vômitos e evoluiu para óbito um mês após o diagnóstico.

\section{DISCUSSÃO}

Aproximadamente 3 a 5\% dos pacientes com neoplasia apresentam sítio primário desconhecido 3 .

Há publicações de tumores primários de cólon que apresentam metástases para pâncreas ${ }^{4,5,6}$ e para colo uterino ${ }^{7}$.

Não foi encontrado caso semelhante a este na literatura. Praderi et $\mathrm{al}^{8}$ apresentaram um caso de paciente que foi submetida a duodenopancreatectomia e sete meses após foi reoperada por adenocarcinoma de reto, mas não há relato sobre a mesma origem dos tumores.

Carcinoma de pâncreas é pouco freqüente na população, mas apresenta mortalidade elevada. $\mathrm{Na}$ América do Norte é a quarta causa de morte ${ }^{9}$ por neoplasia maligna e na Itália a quinta ${ }^{10}$. No Brasil a incidência dessa neoplasia é baixa, mas está entre a $9^{a}$ (no sexo feminino) e $11^{\mathrm{a}}$ causa de morte (no sexo masculino $)^{11}$. A sobrevida média é menor que seis meses. Sobrevivem por cinco anos, dependendo da referência, $10 \%^{12}$ a $1 \%^{13}$ dos pacientes. A maioria dos adenocarcinomas de pâncreas apresenta metástases à distância na data do diagnóstico ${ }^{9}$, quando não são mais ressecáveis ${ }^{10,12}$. Freqüentemente ocorre também invasão por contigüidade. Há alguns relatos de tumores pancreáticos com metástases à distância, para sítios pouco comuns, como região umbilical ${ }^{14}$ e crânio ${ }^{15}$.

s neoplasias de pâncreas não apresentam sintomas iniciais específicos e freqüentemente são ignorados pelo paciente e pelo médico. Dor abdominal vaga, flatulência, vômitos, alteração do hábito intestinal. Tumores de corpo e cauda podem apresentar icterícia numa fase tardia. Radiação da dor para o dorso indica invasão de plexo nervoso esplânico e a perda de peso é multifatorial e ocorre em fase avançada ${ }^{16}$. Sintomas secundários à invasão de outros órgãos ocorrem com certa freqüência. Bringel et a ${ }^{17}$ descreveram um caso 


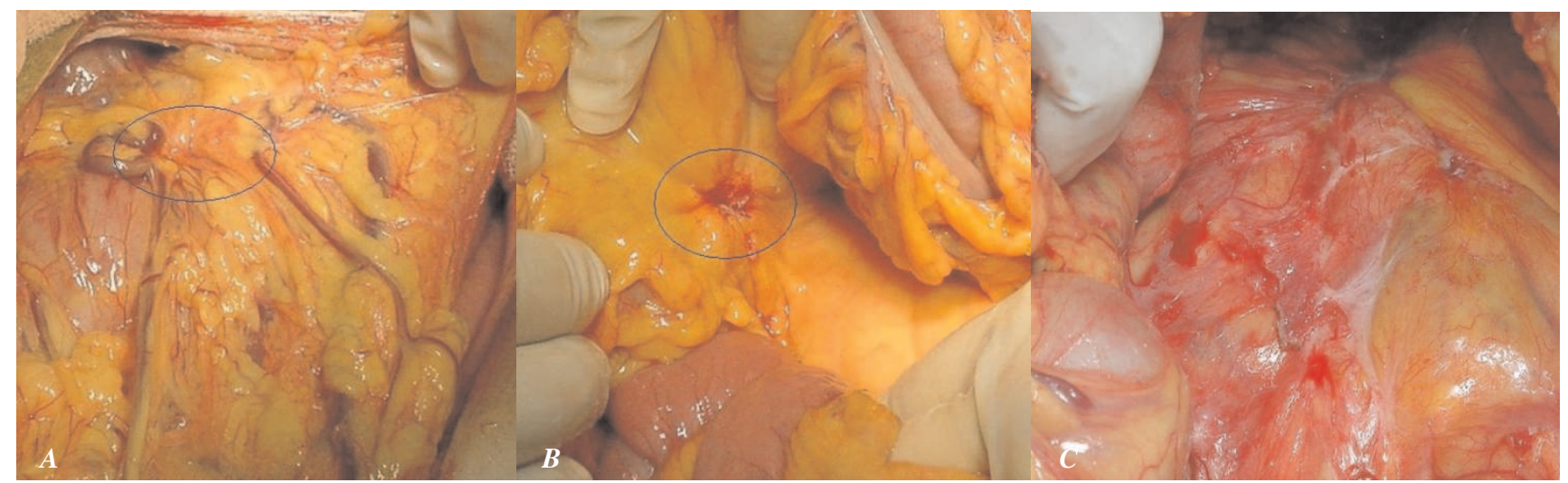

Figura 1 - Foto panorâmica da cavidade peritonial mostrando. A: área de retração do grande omento sobre o tumor pancreático; B: área de invasão do mesocólon transverso; C: aspecto normal do peritônio que recobria o pâncreas após a colectomia direita.

de carcinoma de pâncreas cuja apresentação clínica foi hemorragia digestiva alta por invasão tumoral na parede posterior gástrica.

Diabetes mellitus de início abrupto é relatado em $24 \%{ }^{18}$ a $40 \%{ }^{19}$ dos pacientes e pode ser o primeiro indício de neoplasia de pâncreas. Quando o paciente apresenta diabetes mellitus há mais de três anos não existe associação com a neoplasia e diabetes não é fator de risco para carcinoma de pâncreas. ${ }^{20}$

Alguns fatores contribuíram para o retardo do diagnóstico neste paciente.

Este apresentou diabetes, sem antecedentes pessoais ou familiares, e isso não foi valorizado porque, freqüentemente pacientes idosos pré-diabéticos desenvolvem hiperglicemia durante a internação, por alterações metabólicas secundárias a processo infeccioso. Colecistolitíase com possível empiema foi a suspeita que resultou em colecistectomia de urgência.

Ao dar entrada nesse serviço, uma das hipóteses diagnósticas iniciais para a moléstia desse paciente era neoplasia de pâncreas, que foi descartada, após terem sido encontradas as lesões em cólon e do resultado normal do exame de imagem.

O exame de tomografia computadorizada (TC) tem sido considerado o mais útil para diagnóstico de carcinoma de pâncreas ${ }^{9}$. Mas falhou em detectar alterações pancreáticas nesse caso, retardando o diagnóstico etiológico. Tutton et $\mathrm{al}^{4}$ relataram metástase pancreática de neoplasia de cólon cujo diagnóstico só foi obtido após a realização de PET-Scan (TC com emissão de pósitron). Infelizmente a maioria dos serviços médicos do nosso meio não dispõe desse exame.

A visão da cavidade peritonial não trouxe subsídios para o diagnóstico.Não havia carcinomatose, somente tecido endurecido à palpação e duas lesões planas de cor esbranquiçada em diafragma,nas alças acometidas pelo tumor e aderências, conforme mostra as fig. 1,2, 3 .

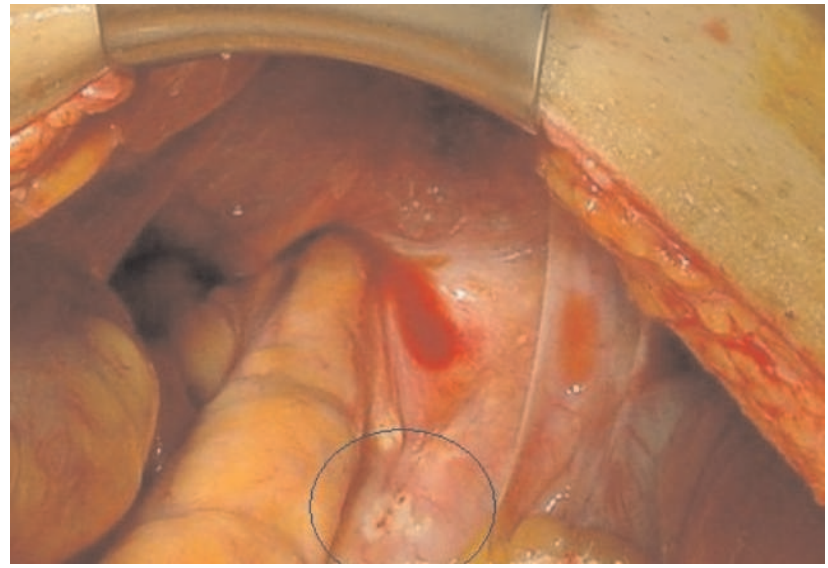

Figura 2 - Foto mostrando a metástase do tumor pancreático em diafragma.

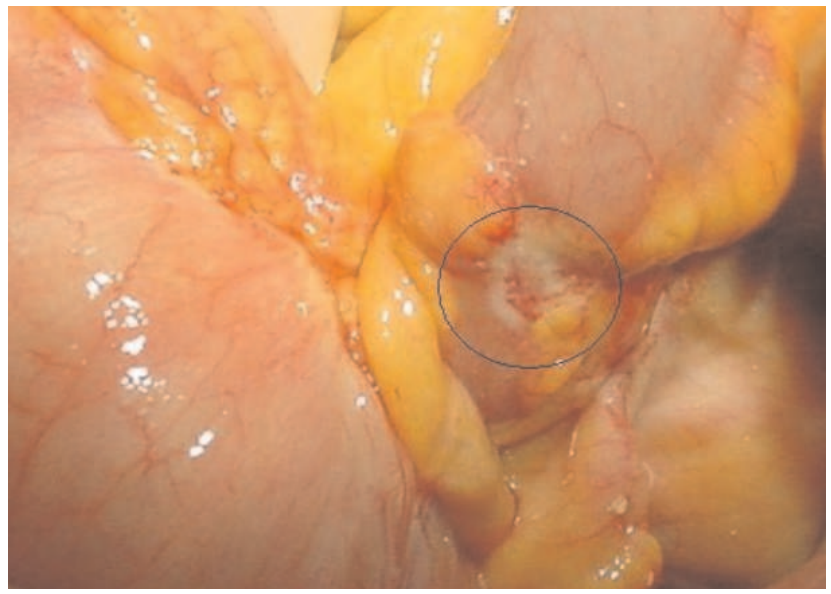

Figura 3 - Foto mostrando a metástase do tumor pancreático em íleo terminal. 


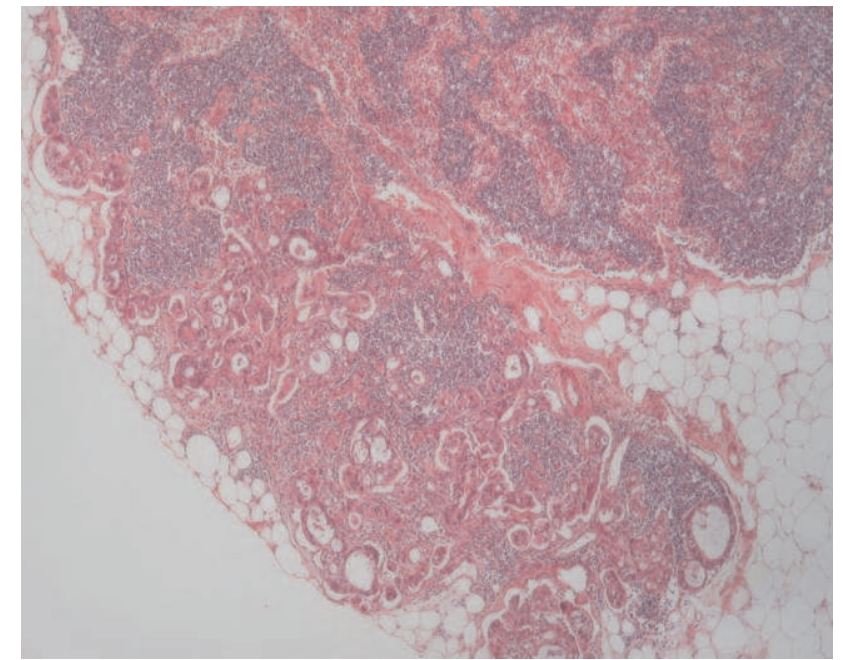

Figura 4 - Microfotografia de tecido linfonodal parcialmente infiltrado na periferia por adenocarcinoma de padrão tubular. (HE $100 x$ ).

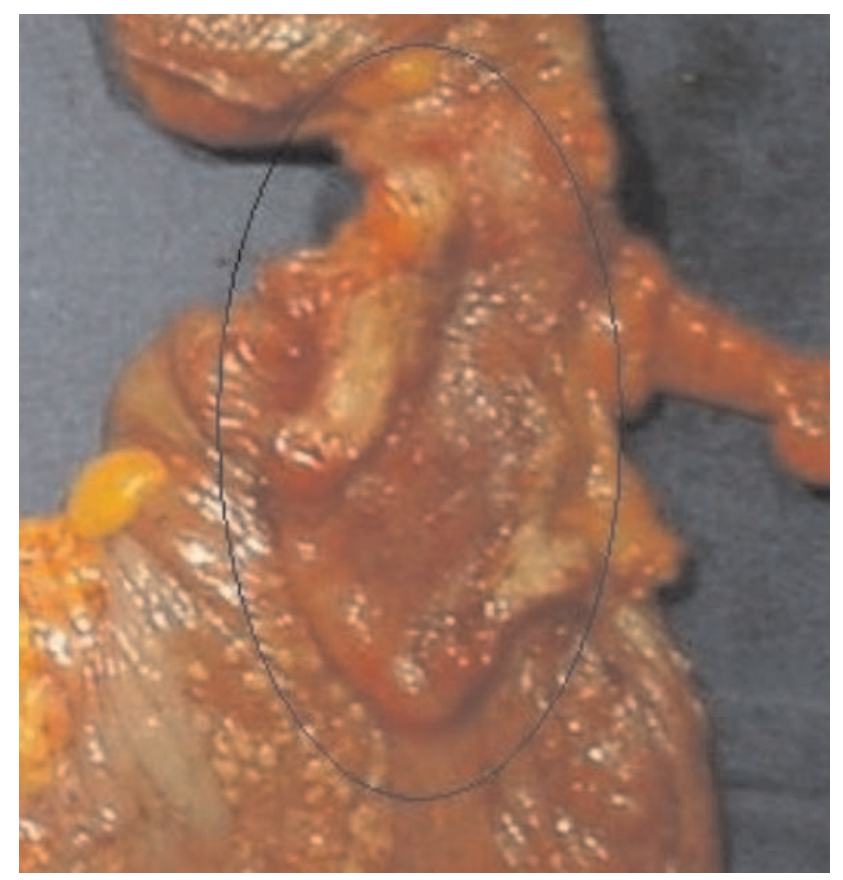

Figura 6 - Foto de peça cirúrgica aberta, produto da colectomia direita, mostrando o aspecto de rigidez e espessamento do íleo terminal e válvula ileocecal.

O exame anatomopatológico pode falhar no diagnóstico de tumor maligno. Quando negativo após a segunda coleta de material feita por profissional experiente, deve-se investigar tumores de origem muscular, estromal, metástases por implante ou outras moléstias.

Neste caso a presença de infiltração periférica de linfonodo por adenocarcinoma tubular (fig.4) não

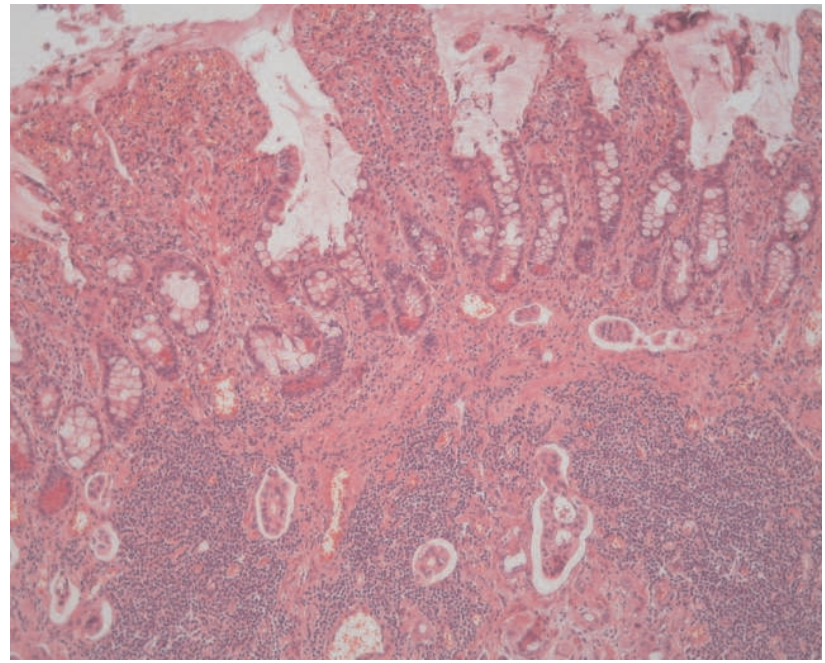

Figura 5 - Microfotografia mostrando infiltração até submucosa por adenocarcinoma tubular. Há nítida resposta desmoplásica (HE $100 x$ ).

ajudou no diagnóstico do tumor primário. A metástase pode ser pouco ou bem diferenciada e ter comportamento diferente do tumor primário. Nas figuras seguintes, correspondentes a fragmentos de parede intestinal com tumor, observa-se que a infiltração tumoral ocorreu da serosa para a submucosa, onde há invasão angiolinfática, mas o epitélio superficial é normal, apresenta só inflamação, provavelmente pela compressão tumoral. A nítida resposta desmoplásica vista na fig.5 provoca perda de distensibilidade da parede e estenose, o que ficou bem evidente no exame macroscópico (fig 6). A falta de células tumorais na mucosa esclareceu o motivo da ausência de tumor nas biópsias realizadas.

O exame por imuno-histoquímica torna possível definir a origem de tumores metastáticos distantes do tumor primário ${ }^{21}$.

Neste caso a CK 7 e 19 positivas indicaram tratar-se de adenocarcinoma de pâncreas, já que no cólon a CK7 é negativa. O CA19-9, usualmente é um marcador para pâncreas. EMA, usadas para avaliar o grau de proliferação do Ca pancreático, e o CEA são positivos em diversas neoplasias e não foram valiosos para elucidação da origem tumoral.

O estudo deste caso mostra que a hipótese diagnóstica, baseada no quadro clínico, não pode ser completamente descartada quando o exame de imagem está normal, sendo necessário em alguns casos, realizar exames mais sofisticados. Quando a origem do tumor primário não está definida é essencial o exame imuno-histoquímico para firmar o diagnóstico. 
Rev bras Coloproct Julho/Setembro, 2007
Sandra Pedroso de Moraes e Cols.

ABSTRACT: OBJECTIVE: Report a case of a rare pancreatic adenocarcinoma presented as synchronic colorectal tumor. CASE REPORT: Seventy six year old man with high intensity and diffuse abdominal pain, diarrhea and vomiting during seven days. At that moment he had been in treatment for gastritis for 2 years and in the last four months he presented hyporexia and weight loss. He was dehydrated and malnourished, with abdominal distention and diffuse abdominal pain on clinical examination. Laboratorial exams showed hyperglycemia, $x$-ray revealed intense abdominal distention, abdominal ultrasound revealed cholecystolithiasis and upper digestive endoscopy showed pangastritis. The computed tomography just confirmed the cholecystolithiasis. A colonoscopy was performed and revealed three tumors located in the rectum, transverse colon and ileocecal valve that were biopsed and just showed inflammatory cells. As the symptoms persist he underwent laparoscopic cholecystectomy that was converted to the open technique when it was observed white flat lesions in the diaphragm peritoneum, the biopsies revealed adenocarcinoma. In the fifth postoperatory day the patient developed obstructive symptoms and underwent right colectomy with double terminal colostomy and pancreas biopsy that showed adenocarcinoma with immunohistochemical profile proving the pancreas as the source. The patient died within one month. CONCLUSION: The normal findings in radiological exams do not dismiss a diagnostic hypothesis and when the source of a tumor is not well established the clinical patterns should be considered and the immunohistochemical profile is essential to confirm the diagnosis.

Key words: pancreatic neoplasms, colorectal neoplasms, hereditary nonpolyposis, neoplasm metastasis, adenocarcinoma, tomography, $\mathrm{x}$ ray computed.

\section{REFERÊNCIAS}

1. Valero J, Regnasco S, Gonzalez O, Doldan I, Sautu de la Riestra A, Kogan Z. Synchronous lesions in advanced colorectal câncer. Acta Gastroenterol Latinoam. 1993; 23(3):165-73.

2. Leon-Rodriguez E, Hernandez MC. Cancer of the colon in the National Institute of Nutrition. II. Synchronous and metachronous tumors. Rev Invest Clin 1996; 48(4):275-9.

3. Berglund A, Nygren P, Hagberg H, Pahlman L, Sundin A, Sundstrom C. Limit investigation in cancer of unknown primary site. Lakartidningen 2005; 102(41):2946-8, 2950-2.

4. Tutton MG; George M; Hill ME; Abulafi AM. Solitary pancreatic metastasis from a primary colonic tumor detected by PET scan: report of a case. Dis Colon Rectum 2001; 44(2):288-90.

5. Pereira-Lima JC, Coral GP, Bayer LR, da Silva CP. Metastasis from colon carcinoma in the dorsal pancreas of a patient with pancreas divisum: report of a case. Hepatogastroenterology. 2000; 47(32):554-5.

6. Charnsangavej C, Whitley NO. Metastases to the pancreas and peripancreatic lymph nodes from carcinoma of the right side of the colon: CT findings in 12 patients. AJR Am J Roentgenol.; 1993; 160(1):49-52.

7. Belloso, Rafael M; Miquilarena, Rodolfo E; Ayala N., Luis A; Zucker, Eva; Souchon, Eduardo; Apeloig, Paulina Altaras de. Carcinoma de colon metastasico a cuello uterino: revision de metastasis inusuales y presentacion de un caso. GEN 1990;44(1):63-6.

8. Praderi, R. C; Harretche, M; Estefan, A; Parodi, H; Hernández, W; Boudrandi, Sonia; Navarrete, H. Resecciones simultáneas o sucesivas de páncreas y colon por tumores de ambos órganos, carcinomas de colon infiltrando duodeno y de páncreas caudal invadiendo colon izquierdo: a propósito de diez casos. Acta gastroenterol. latinoam 1999; 29(3):95-9.

9. Freelove R; Walling AD. Pancreatic cancer: diagnosis and management. Am Fam Physician 2006; 73(3):485-92.

10. Chiaro MD, Boggi U, Presciuttini S, Bertacca L, Croce C, Mosca I, Mosca F. Genetics of Pancreatic Câncer: whereare we now? Where are we going? J Pancreas 2005; 6(1):607.

11. CÂNCER - Epidemiologia, informação e vigilância. Atlas de mortalidade 1979-1999. In: INSTITUTO NACIONAL DO CÂNCER. Disponível em : <http:www.inca.gov.br

12. Bornman PC, Beckingham IJ. Pancreatic tumours: clinical review. BMJ 2001; 322: 721-3.

13. Keleg S, Büchler P, Ludwig R, Büchler MW, Friess H. Invasion and metastasis in pancreatic cancer: review. Molecular Cancer 2003; 2:14.

14. Crescentini, Fábio; Deutsch, Fernanda; Sobrado, Carlos Walter; Araújo, Sérgio de. Umbilical mass as the sole presenting symptom of pancreatic cancer: a case report. Rev. Hosp. Clin. Fac. Med. Univ. Säo Paulo 2004; 59(4):198-202.

15. Aydin MV; Cekinmez M; Kizilkilic O; Kayaselcuk F; Sen O; Altinors N. Unusual case of skull metastasis secondary to pancreatic adenocarcinoma. Pathol Oncol Res 2005; 11(3):1823.

16. Takhar AS, Palaniappan P, Dhingsa R, Lobo DN. Recent developments in diagnosis of pancreatic cancer: clinical review. BMJ 2004; 329:668-73.

17. Bringel RWA, Souza CPM, Araujo SEA, Lopasso FP, Gama Rodrigues JJ, Pinotti HW, Laudanna AA. Squamous cell carcinoma of the pâncreas with gastric metastasis: case report. Rev. Hosp. Clin. Fac. Méd. Univ. São Paulo 1996; 51(5):1957. 
18. Carneiro BGMC, Petroianu A, Rodrigues FHOC, Rocha RF, Liu RP, Orsi VL. Avaliacäo do câncer de pâncreas no Hospital das Clínicas da Universidade Federal de Minas Gerais. An. Paul. Med. Cir 2002; 129(2):28-35

19. DiMagno EP. Pancreatic cancer: clinical presentation, pitfalls and early clues. Ann Oncol 1999;10 Suppl 4:140-2

20. Gullo L. Diabetes and the risk of pancreatic cancer. Ann Oncol 1999;10 Suppl 4:79-81

21. Marzán L A R, Pupo Neto J A, Bottino, A M C F, Lacombe, D L P. Análise crítica da classificaçäo e estudo imuno- histoquímico dos tumores da camada muscular do cólon e do reto: revisäo de 11 casos. Rev. Bras Coloproct 2003;23(4):244255.

\section{Endereço para correspondência:} SANDRA PEDROSO DE MORAES

Rua Barão Geraldo de Rezende, 282, conj. 53

Fax: (19) 32360436

E-mail: spmoraes@metrosat.com.br 